\title{
Implementation and Comparison of Lattice Module and Discontinuity Factors Module in PROTEUS Toolsets
}

\author{
Kan $\mathrm{Ni}^{1}$, Jason Hou ${ }^{1}$, and Maria Avramova ${ }^{1}$ \\ ${ }^{1}$ North Carolina State University \\ 2500 Stinson Drive, Raleigh, NC 27695-7909
}

kni@ncsu.edu, jason.hou@ncsu.edu,mnavramo@ncsu.edu

\begin{abstract}
State-of-the-art core nodal diffusion calculation involves the use of assembly discontinuity factor (ADF) to improve computational accuracy by introducing degree of freedom describing the relationship between interfacial discontinuities in nodal calculation [1]. The form of ADF known as the Flux based ADF (FDF) generated from flux information is recommended in the conventional two-level core calculation scheme. The multi-group cross-sections were generated using SCALE 6.2 NEWT and verified with KENO-VI [2]. A lattice module has been created for the high-fidelity neutron transport code MOCEX [3] to generate the group constants and side-independent ADFs. This new capability is verified against the reference code SCALE 6.2 NEWT under both serial and parallel modes. The implementation of ADF is performed in this work and further verified by comparing core $k_{\text {eff. }}$ The calculation results show that the newly implemented ADF module consistently improved the accuracy of the PROTEUS-NODAL (NODAL) diffusion solver, which will become an affordable candidate for the following research of High-to-Low (Hi2Lo) transport-to-diffusion informing scheme [4].
\end{abstract}

KEYWORDS: Discontinuity factor; PROTEUS; Nodal method; NEAMS

\section{INTRODUCTION}

Nodal methods were developed to perform large scale reactor calculations in an efficient way, while still maintaining certain level of accuracy. This is achieved through the homogenization of information in a relatively large spatial region so that reactor simulation could be conducted with a reduced mesh number [5]. The reactor core calculation could be further represented by the conventional two-step approach. A lattice calculation considering the detailed neutron flux in assembly with reflective boundary condition is performed as the first step, to homogenize and condense region-based cross-sections into few group macroscopic cross sections. The core simulation is then performed by nodal code with the generated assembly macroscopic cross-sections. This method works well without ADF in conventional Light Water Reactor (LWR) modeling but could be problematic when applied to more complicated and highly heterogeneous reactor core designs. The ADF is therefore recommended to provide additional information to the preserved the low-order information [6] in highly heterogeneous reactor core calculations.

PROTEUS is a code package under development of NEAMS project, which includes three different neutronics solvers: SN (discrete ordinates), MOC (method of characteristics) and NODAL (nodal transport) [3]. PROTEUS-MOC (MOCEX) is used in this study to generate the whole core reference solution. However, MOCEX hasn't been integrated with a cross-section homogenization and ADF generation module. In order to establish the accessibility for the conventional approach inside of PROTEUS code 
between MOCEX and NODAL, a lattice module that is able to homogenize the assembly cross-sections will be introduced to the MOCEX in this study.

The variational nodal transport code NODAL was recently added into the PROTEUS code suite. Two solution options have been developed including the diffusion theory (P1) and a nodal simplified $\mathrm{P}_{3}(\mathrm{SP} 3)$. The $\mathrm{P} 1$ option of this code is developed based on the DIF3D-VARIANT $\mathrm{P}_{\mathrm{N}}$ transport methodology. The implementation a new 2D Cartesian Geometry ADF option to the NODAL P1 solver is performed in this study.

This paper will cover several modifications added to the PROTEUS code including the development of the lattice module and the implementation of the ADF capability. The performance of each newly implemented module is assessed by various code-to-code verification.

\section{C5G7 BENCHMARK PROBLEM}

The numerical case for verification is a modification of the well-studied heterogeneous C5G7 benchmark problem. The core configuration and material composition can be found in [7]. Different from the original C5G7 benchmark, the revised C5G7 benchmark preserves the heterogeneous geometry of the fuel pin but removes the outer aluminum cladding. The revised C5G7 benchmark also contains 8 unique materials: three enrichments of MOX fuel, $\mathrm{UO}_{2}$ fuel, aluminum cladding for guide tubes and fission chamber, inner instrument chamber and moderator. The $\mathrm{UO}_{2}$ fuel assemblies are located in the core center and corresponding diagonal positions, while the rest of the fuel region are filled with MOX assembly. The modified pin cell geometries are depicted in Figure 1.
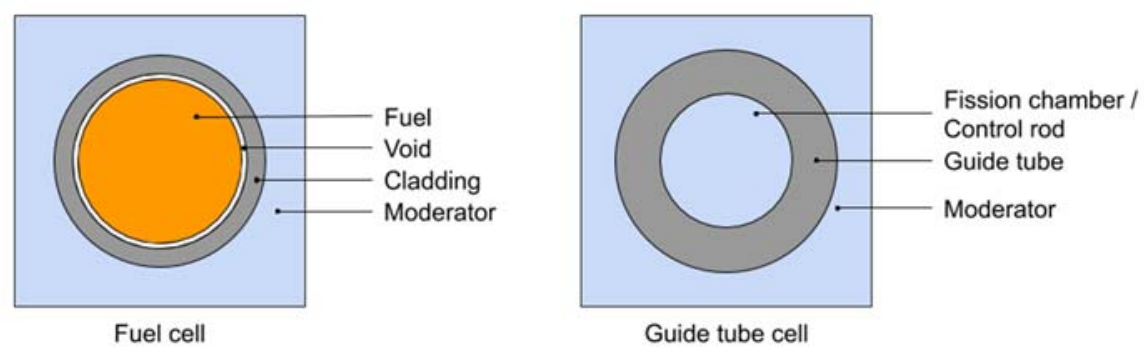

Figure 1. Modified pin cell layout

\section{METHODOLOGY DEVELOPMENT AND IMPLEMENTATION}

In the conventional two-step approach, a detailed flux calculation using a lattice code is needed to homogenize the assembly-based cross-section and to generate the discontinuity factor. The cross-section set with higher order anisotropic scattering blocks for high-fidelity calculation cross-section homogenization module for MOCEX are developed in this study. This section will cover the generation of macroscopic cross-section library, homogenization of the assembly cross-section, ADF generation and ADF implementation. The verification of each module will be provided.

\subsection{Cross-section Library Generation}

The SCALE 6.2 code package is a widely used simulation and modeling suite for reactor physics analysis and has been integrated into DOE Nuclear Energy Advanced Modeling and Simulation (NEAMS) workbench, which makes it easier to pass information to the PROTEUS, also onboard NEAMS. From the SCALE 6.2 toolset, the deterministic lattice code NEWT is selected as the candidate for macroscopic multi- 
group cross-section libraries calculation. The quality of the multi-group macroscopic cross-section is evaluated by the Monte Carlo code KENO-VI.

The SCALE system provides an ENDF/B-VII.1 based 252-group cross-sections library that is suitable for thermal reactor calculation [2]. A new set of 18-group macroscopic multi-group cross-section library that suitable for high-fidelity LWR calculation [8] is generated using NEWT based on the aforementioned 252group library. The energy structure for the 18-group macroscopic cross-section set is shown in Table I.

Table I. 18-group structure used in the study

\begin{tabular}{|c|c|c|c|c|c|}
\hline $\begin{array}{c}\text { Group } \\
\text { Index }\end{array}$ & $\begin{array}{c}\text { Upper Group } \\
\text { Limit (eV) }\end{array}$ & $\begin{array}{c}\text { Group } \\
\text { Index }\end{array}$ & $\begin{array}{c}\text { Upper Group } \\
\text { Limit }(\mathrm{eV})\end{array}$ & $\begin{array}{c}\text { Group } \\
\text { Index }\end{array}$ & $\begin{array}{c}\text { Upper Group } \\
\text { Limit }(\mathrm{eV})\end{array}$ \\
\hline 1 & $2.00 \mathrm{E}+07$ & 7 & $1.70 \mathrm{E}+04$ & 13 & $3.00 \mathrm{E}+00$ \\
\hline 2 & $3.00 \mathrm{E}+06$ & 8 & $3.00 \mathrm{E}+03$ & 14 & $1.00 \mathrm{E}+00$ \\
\hline 3 & $1.40 \mathrm{E}+06$ & 9 & $5.50 \mathrm{E}+02$ & 15 & $4.00 \mathrm{E}-01$ \\
\hline 4 & $9.00 \mathrm{E}+05$ & 10 & $1.01 \mathrm{E}+02$ & 16 & $1.00 \mathrm{E}-01$ \\
\hline 5 & $4.00 \mathrm{E}+05$ & 11 & $3.00 \mathrm{E}+01$ & 17 & $5.00 \mathrm{E}-02$ \\
\hline 6 & $1.00 \mathrm{E}+05$ & 12 & $1.00 \mathrm{E}+01$ & 18 & $1.00 \mathrm{E}-02$ \\
\hline
\end{tabular}

Region-based macroscopic cross-section is generated for $\mathrm{UO}_{2}$ and MOX assemblies with reflective boundary condition. Multi-group cross-sections contain anisotropic scattering blocks with Legendre expansion order up to 5 and thus satisfies the needs of high-fidelity calculation.

Whole-core criticality calculations were performed using the Monte Carlo code KENO-VI with the 252group and 18-group cross-section libraries, respectively. The comparison of the calculation results of the C5G7 benchmark problem represents the effect of group condensation from 252-group to 18-group. It is observed that the group condensation introduces a small eigenvalue difference of $79.5 \mathrm{pcm}$ to the $\mathrm{C} 5 \mathrm{G} 7$ whole core. As depicted in Figure 2, a Fission Rate Density (FRD) difference within the range of $-3.21 \%$ to $1.61 \%$ is observed during the condensation procedure, while Root Mean Squared (RMS) value along all the differences in each fuel pin cell is calculated as $0.68 \%$, featuring a stable difference distribution in the fuel region. This macroscopic cross-section set will be used in the following PROTEUS calculation.

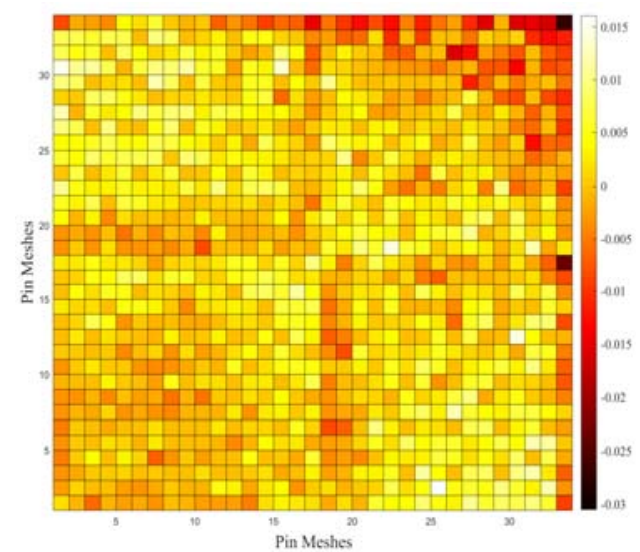

Figure 2. Distribution of pin FRD difference of C5G7 benchmark fuel region during group condensation 


\subsection{Implementation of Lattice Physics Capability in MOCEX}

MOCEX is a high-fidelity solver based on the 2D/3D MOC formulation [9]. Current study needs a homogenized assembly cross-section for low-order solver NODAL and the future Hi2Lo informing scheme inside of PROTEUS toolsets. Thus, a lattice module able to homogenize region-based macroscopic crosssection into assembly averaged cross-section is created for MOCEX, and this lattice module will also work as a bridge in the future Hi2Lo informing scheme inside the PROTEUS toolsets.

\subsubsection{Generation of equivalence parameter}

In the lattice module created for MOCEX, the assembly cross-sections are homogenized based on the fluxvolume weighting to preserve the reaction rates of the heterogeneous domain by using the detailed flux obtained from fine mesh calculation. A coarse mesh file is provided to MOCEX, and MOCEX will use the fine mesh flux to homogenize the cross-sections in the regions defined by the coarse mesh file. As the MOCEX is a well-developed parallel code that runs in angular, space and energy group decomposition, the homogenization module will also be extended for parallel execution for time-saving purpose. For the targeted homogenization region $A$, the homogenized cross-section in group $g$ of the reaction type $x$ is given by

$$
\Sigma_{x, g}=\frac{\sum_{i \in A} V_{i} \phi_{i, g} \Sigma_{x, g}^{i}}{\sum_{i \in A} V_{i} \phi_{i, g}}
$$

where $V_{i}, \phi_{i, g}$ and $\Sigma_{x, g}^{i}$ are the volume, the average flux and cross-sections in fine mesh $i$ of homogenization region $A$. Thus, the homogenized assembly cross-section can be calculated by placing calculation domain to a single assembly.

FDF can be produced in the lattice module to preserve the interface flux discontinuity information. Based on Generalized Equivalence Theory (GET) [1], the FDF is obtained for each surface of the assembly, calculated by the ratio of the surface-averaged flux by heterogeneous solver to the surface-averaged flux by homogeneous solver [10]. As the current two-step calculation is based on single assembly calculation model with reflective boundary condition, the surface-average flux computed from low-order homogenous solver is the same as the assembly average flux. For a specific surface $s$, the $\operatorname{FDF} f_{g}^{s}$ is defined as

$$
f_{g}^{s}=\frac{\bar{\phi}_{g}^{s, h e t}}{\bar{\phi}_{g}^{s, h o m}}=\frac{\bar{\phi}_{g}^{s, h e t}}{\sum_{A} V_{i} \phi_{i, g}}
$$

\subsubsection{Verification of lattice physics capabilities}

Two verification processes, namely internal correctness verification (Case 1 vs. 2) and code-to-code verification (Case 2 vs. 3) are performed separately, as shown in Figure 3. The lattice module also enables the ability to generate FDFs needed in NODAL calculation, a group to group verification is also conducted comparing through comparison with FDFs generated from NEWT. 


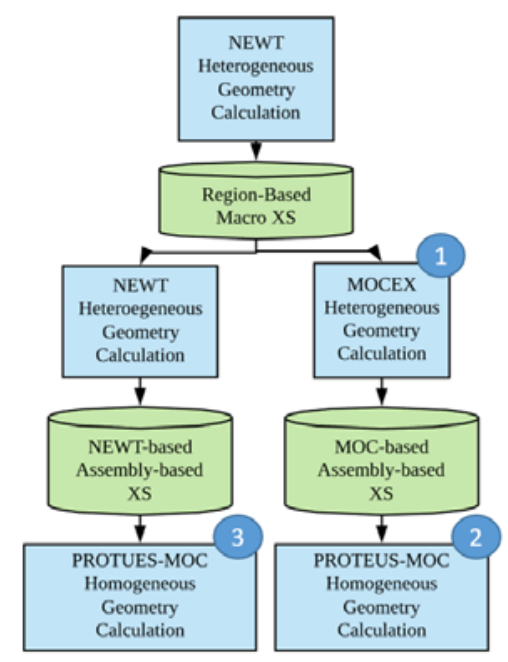

Figure 3. Verification process for MOCEX lattice ability

Internal correctness verification (Case 1 vs. Case 2) is performed to verify the correctness of the crosssections homogenization capability in both serial and parallel running modes. As shown in row 3 in Table II, this procedure is conducted on C5G7 benchmark by comparing the eigenvalue result of homogenized cross-section with the result using original cross-section. An acceptable minor eigenvalue difference less than $1 \mathrm{pcm}$ for two assemblies in both sequential and parallel running mode has been observed, which indicates that the homogenized cross-sections successfully preserve the needed low-order information for nodal calculation.

The code-to-code verification procedure (Case 2 vs. Case 3) accesses the cross-sections homogenization capability of single assembly in MOCEX versus the reference lattice code NEWT and the comparison result is shown on row 4 of Table II. A small eigenvalue difference less than $35 \mathrm{pcm}$ is observed indicating the lattice module could perform a reliable cross-section homogenization process against the well verified reference code NEWT. From the result of two comparison, the new MOCEX lattice module is ready to be applied for cross-sections homogenization for the NODAL solver.

Table II. Verifications of MOCEX lattice module

\begin{tabular}{|c|c|c|c|c|c|}
\hline MOX Assembly & $\begin{array}{c}\text { Serial } \\
\text { Running }\end{array}$ & $\begin{array}{c}\text { Parallel } \\
\text { Running }\end{array}$ & $\mathrm{UO}_{2}$ Assembly & $\begin{array}{c}\text { Serial } \\
\text { Running }\end{array}$ & $\begin{array}{c}\text { Parallel } \\
\text { Running }\end{array}$ \\
\hline $\begin{array}{c}\text { Case } 1 \\
\left.\text { (Ref. } k_{\text {eff }}\right)\end{array}$ & 1.21906 & 1.21906 & $\begin{array}{c}\text { Case 1 } \\
\left.\text { (Ref. } k_{\text {eff }}\right)\end{array}$ & 1.34961 & 1.34962 \\
\hline $\begin{array}{c}\text { Case 1 vs. Case } 2 \\
\text { (pcm) }\end{array}$ & -0.1 & 0.3 & $\begin{array}{c}\text { Case 1 vs. Case 2 } \\
(\mathrm{pcm})\end{array}$ & 0.4 & 0.1 \\
\hline $\begin{array}{c}\text { Case 2 vs. Case } 3 \\
\text { (pcm) }\end{array}$ & \multicolumn{2}{|c|}{-10.6} & $\begin{array}{c}\text { Case 2 vs. Case 3 } \\
(\mathrm{pcm})\end{array}$ & \multicolumn{2}{|c|}{-33.2} \\
\hline
\end{tabular}

The FDF generation module implemented in the MOCEX is verified by comparing the generated FDFs against the ones from NEWT. FDFs are generated for two fuel assemblies using both MOCEX and NEWT, an absolute maximum relative difference of $3.21 \%$ and an averaged absolute difference of $0.08 \%$ are obtained when comparing the group-wise FDFs, which indicates the successfully implementation of FDF generation function inside of lattice module. 


\subsection{Implementation of ADF Module in NODAL}

It is realized that the use of ADFs is crucial in the NODAL code for preserving information from highorder solver to retain the accuracy of full core simulation result. Moreover, the implementation of ADF module is also required in the future Hi2Lo iteration calculations.

To implement ADF in NODAL, the continuity of the outgoing partial currents of the target node is replaced with the incoming current of the target node and the outgoing current of the last node in the within group sweeping process. This process introduces discontinuity to the partial current on assembly interface based on Lawrence's approach [5] using Eq. (3) and Eq. (4).

where

$$
\alpha J_{g x+}^{i n, k}=\left(\frac{1}{1-\alpha}\right)\left(J_{g x-}^{o u t, l}+\alpha J_{g x+}^{o u t, k}\right)
$$

$$
\alpha \equiv \frac{1}{2}\left(1-\frac{f_{g x+}^{k}}{f_{g x-}^{l}}\right)
$$

and $f_{g x+}^{k}$ and $f_{g x-}^{l}$ are the FDFs at the right and left inter-surface of two neighboring nodes $\mathrm{k}$ and 1 , respectively. $J_{g x-}^{o u t, l}$ and $J_{g x+}^{\text {out }, k}$ represent the outgoing partial current on the left inter-surface of right node $l$ and the right inter-surface left node $k$, respectively. $J_{g x+}^{i n, k}$ denotes the incoming partial current of the right inter-surface of left node, as depicted in Figure 4. Code modification has been made in the red-black iteration part of the original NODAL solver, enabling the correction of the partial current passing between nodes.

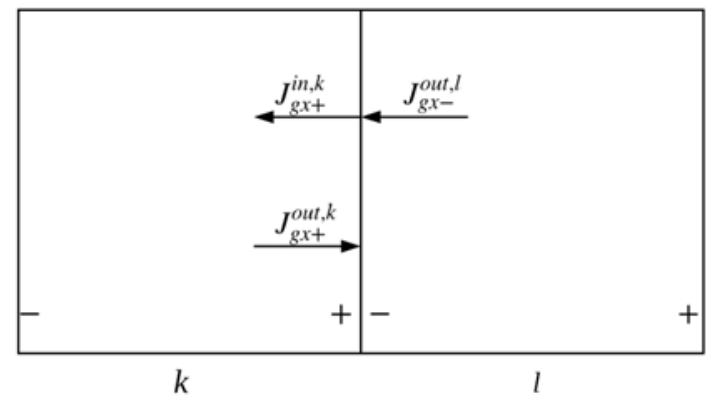

Figure 4. Scheme of partial current flow in the two adjacent nodes

In this verification process, the fully heterogeneous core calculation results from MOCEX P1 solver are regarded as references. The following test cases with different core configurations have been designed to verify the implementation of ADF:

a) Modified C5G7 core without reflector, with reflective boundary condition;

b) Modified C5G7 core;

c) Modified KAIST quarter core Problem $1 \mathrm{~B}$ with 5 by $5 \mathrm{MOX} \& \mathrm{UO}_{2}$ assemblies. The baffle is not included in the calculation.

The newly implemented ADF module is verified by comparing the NODAL calculation result with/without ADF to the references. All the NODAL calculation cases will use the out-scatter transport corrected total cross-sections generated by the lattice module in MOCEX. The calculated core eigenvalue for those four cases are summarized in Table III. 
Table III. ADF Pprformance in $k_{\text {eff }}$ of NODAL solver

\begin{tabular}{|c|c|c|c|}
\hline a) Modified C5G7 core without reflector & \multicolumn{3}{|c|}{} \\
\hline \multirow{2}{*}{ MOCEX } & \multicolumn{2}{|c|}{1.28690} & Difference (pcm) \\
\hline \multirow{2}{*}{ NODAL } & without ADF & 1.28132 & -558.1 \\
\cline { 2 - 4 } & with ADF & 1.28194 & -496.0 \\
\hline b) Modified C5G7 core & \multicolumn{3}{|c|}{} \\
\hline MOCEX & 1.21245 & Difference (pcm) \\
\hline \multirow{2}{*}{ NODAL } & without ADF & 1.19473 & -1772.3 \\
\cline { 2 - 4 } & with ADF & 1.20055 & -1190.0 \\
\hline c) Modified KAIST benchmark 1B & \multicolumn{3}{|c}{} \\
\hline MOCEX & 1.28552 & Difference (pcm) \\
\hline \multirow{2}{*}{ NODAL } & without ADF & 1.28041 & -511.0 \\
\cline { 2 - 4 } & with ADF & 1.28138 & -413.4 \\
\hline
\end{tabular}

As shown in Table III, the use of ADF consistently improves the accuracy of core eigenvalues by $\sim 60 \mathrm{pcm}$ (a) to $\sim 600 \mathrm{pcm}$ (b). There is relatively large error in the $\mathrm{C} 5 \mathrm{G} 7$ calculation result, which may come from the highly heterogeneity and non-diffusiveness of its configuration. The error is decreased when it is applied to KAIST 1B core as the core dimension is much larger. From the result, the consistent improvement indicates that information carried by the FDF could inform NODAL to correct its partial current sweeping information. The implemented ADF module can improve the performance of conventional two-steps whole core calculation approach with limited effort in the code development.

The detailed flux distribution information has also been assessed for the C5G7 benchmark. The normalized flux distribution of MOCEX is selected as the reference. The relative improvement of the ADF implementation to the flux distribution comparing to original NODAL solver in the fuel region is shown in Figure 5. A significant flux distribution improvement from $7 \%$ to $42 \%$ in both MOX assembly is observed for all energy range. However, the ADF implementation introduced some non-negligible errors in UO2 assembly, especially in fast region. This effect may originate from the strong heterogeneity at the adjacent region between $\mathrm{MOX}$ and $\mathrm{UO}_{2}$ assembly. As the fast flux is tended to be distorted between two different assemblies due to long travel distance of fast neutron, using single assembly calculation to generate ADFs cannot capture the spectrum gradient of fast flux well. In comparison, the thermal flux is performing much better in $\mathrm{UO}_{2}$ assembly as the thermal flux distortion region usually within few centimeters and it can be represented by the single assembly calculation.

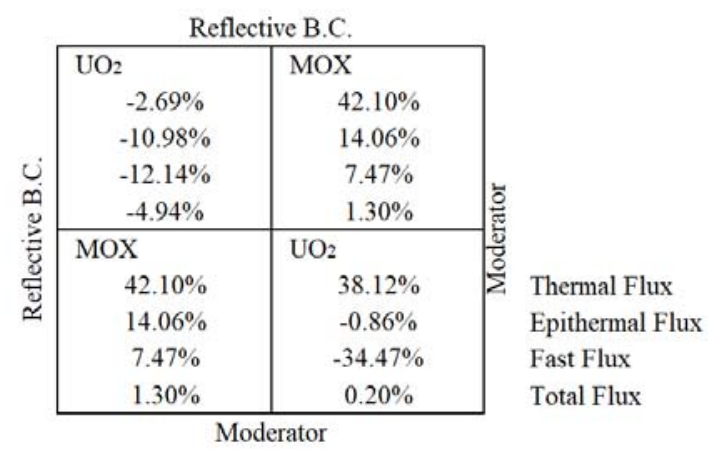

Figure 5. Relative improvement of ADF implementation for flux distribution in modified C5G7 fuel region 


\section{CONCLUSION}

An ADF module has been implemented into NODAL diffusion option to improve the accuracy of the conventional two-step method. This work is established by generating a new set of multi-group crosssection library for $\mathrm{C} 5 \mathrm{G} 7$ benchmark, introducing a lattice module to MOCEX and implementing ADF to NODAL. A series of verifications were performed and consistent improvements of the calculation result using NODAL are observed for several test cases.

However, eigenvalue difference between fully heterogeneous reference calculation of MOCEX and NODAL is still large. This effect may be attributed to the fact that FDFs are generated in single assembly calculation, which doesn't capture all discontinuity from MOCEX. The FDFs generated using whole-core model to catch all available information are recommended to rigorously verify the NODAL. In the future study, color-set method may be involved in the FDFs generation process to improve the NODAL result. This ADF module will also be adapted in the future Hi2Lo informing algorithm between MOCEX and NODAL.

\section{ACKNOWLEDGMENTS}

This research is being performed using funding received from the DOE Office of Nuclear Energy's Nuclear Energy University Programs (NEUP 18-15104). The technical assistance and feedback of experts at the Argonne National Laboratory (ANL) is highly appreciated. In particular, the authors would like to thank Dr. Changho Lee and Dr. Yeon Sang Jung for his assistance.

\section{REFERENCES}

[1] K. Smith, "Assembly Homogenization Techniques For Light Water Reactor Analysis," Progress in Nuclear Energy, vol. 17, no. 3, pp. 303-335, 1986.

[2] B. T. Rearden and M. A. Jessee, Editors, "SCALE Code System," ORNL/TM-2005/39, Oak Ridge, TN, Aug 2016.

[3] C. H. Lee, Y. S. Jung, and M. A. Smith, "FY17 Status Report on NEAMS Neutronics Activities," Nuclear Engineering Division, Argonne National Laboratory, Chicago, Sep, 2017.

[4] B. Ivanov, E. Müller, M. Ouisloumen, K. Ivanov, "Embedded lattice transport calculations based on PARAGON-NEM code system for reactor core analysis," in International Conference on the Physics of Reactors, Interlaken, Switzerland, September 14-19, 2008.

[5] R. D. Lawrence, "Progress in NODAL Methods for the Solution of the Neutron Diffusion and Transport Equations," Progress in Nuclear Energy, vol. 17, no. 3, pp. 271-301, 1986.

[6] R. Sanchez, "Assembly homogenization techniques for core calculations," Progress in Nuclear Energy, vol. 51, pp. 14-31, 2009.

[7] M. D. DeHart, et al, "NEA/NSC OECD/NEA Time Dependent Benchmark using Rattlesnake, Rattlesnake-IQS and TDKENO," Idaho National Laboratory, Idaho Falls, ID, 2016.

[8] S. Langenbuch, A. Seubert, and W. Zwermann, "HIGH ACCURACY LARGE SCALE MONTE CARLO AND DETERMINISTIC TRANSPORT CALCULATIONS FOR CRITICAL SYSTEMS," in $M \& C$, Monterey, California, April 15-19, 2007.

[9] Y. S. Jung, C. H. Lee and M. A. Smith, "Verification of High-Fidelity Neutronics Code PROTEUS for C5G7 Benchmark Problems," in Transactions of the American Nuclear Society, San Fransisco, California, 2017.

[10] J. Hou, H. Choi, K.N. Ivanov, "Development of an iterative diffusion-transport method based on MICROX-2 cross section libraries," Annals of Nuclear Energy, vol. 77, pp. 335-342, 2015. 